\title{
Efecto de la incorporación de pasto estrella sobre el mejoramiento del proceso y la calidad del producto del compostaje de biorresiduos
}

\author{
KEVIN FoRONDA-ZAPATA ${ }^{1}$ \\ Cielo ARIAS-Giraldo ${ }^{1}$ \\ JONATHAN SOTO-PAZ ${ }^{1}$ \\ Luis FERnANdo MARMOLEJo-REBELLÓN ${ }^{1}$ \\ PATRICIA TORRES-LOZADA ${ }^{1}$
}

\section{Resumen}

La predominancia orgánica de biorresiduos de origen municipal (BOM) presentes en residuos sólidos municipales, favorece su aprovechamiento mediante el compostaje; sin embargo, presentan deficiencias fisicoquímicas que pueden ser mitigadas con la incorporación de materiales de soporte (MS). En este estudio se evaluó el efecto de la incorporación de Pasto Estrella (PE) como MS, sobre el compostaje de BOM, evaluando cuatro proporciones BOM:PE (A0-100:00, A1-90:10, A2-80:20, A3-70:30), evidenciándose efectos favorables de las mezclas respecto al tratamiento control (A0 de solo BOM). A2 mantuvo las mayores temperaturas; A2 y A3 registraron la mayor reducción de sólidos volátiles (SV) y concentración final de nitrógeno total (NT). Los productos finales de A2 y A3 presentaron mejor calidad en términos de capacidad de intercambio catiónico y nutrientes (fósforo, potasio y NT), densidad aparente, capacidad de retención de humedad y contenido de materia orgánica, siendo el producto de A2 el de mayor valor agronómico y que mejor se ajusta a la NTC 5167. Proporciones menores o iguales a la de A1 no tienen efecto significativo sobre el proceso y calidad del producto final; proporciones mayores a A3 podrían favorecer la pérdida de NT por aumento en la porosidad, disminuyendo así el valor agrícola del producto.

Palabras Clave: Biorresiduos, Compostaje, Co-compostaje, Material de soporte, Pasto Estrella.

1 Universidad del Valle, Facultad de Ingeniería, Grupo de Investigación Estudio y Control de la Contaminación Ambiental - ECCA, Cali-Colombia.

Autor de correspondencia: Torres-Lozada, P. (Patricia): Universidad del Valle, Facultad de Ingeniería, Grupo de Investigación Estudio y Control de la Contaminación Ambiental - ECCA, Calle 13 \# 100-00, Cali-Colombia. Código Postal: 760000. Teléfono: (57) 2 3312175. Correo electrónico: patricia.torres@correounivalle.edu.co.
Historia del artículo:

Artículo recibido: 26-VIII-2019/ Aprobado: 15-I-2020

Disponible online: 15 de enero de 2020

Discusión abierta hasta septiembre de 2021 


\section{Effect Of Grass Star Incorporation On The Composting Biowaste Process And On The Quality Of The Product}

\section{Abstract}

The predominantly organic composition of biowaste (BW) present in municipal solid wastes, enhances its use through composting; however, these present physicochemical deficiencies that can be mitigated with the incorporation of conditioning materials as the support materials (SM). On this study, it was evaluated the effect of the incorporation of star grass (SG) on BW composting in four BW:SG ratios (A0-100: 00, A1-90: 10, A2-80: 20, A3-70: 30), showing favorable effects with respect to $\mathrm{A} 0(100 \% \mathrm{BOM})$. A2 maintained the highest temperatures; $\mathrm{A} 2$ and $\mathrm{A} 3$ recorded the greatest reduction of volatile solids (VS) and final concentration of total nitrogen (TN). The final products of A2 and A3 also presented better quality in terms of cation exchange capacity, nutrient content (total phosphorus, potassium and nitrogen), bulk density, moisture retention capacity and organic matter content; being A2 product, the material with highest agronomic value, in accordance with Colombian Technical Standard. Proportions less or equal to the one evaluated in A1, do not have a significant effect on the process and quality of the final product and, proportions greater than A3 could favor the loss of nitrogen due to the increase in porosity, thus decreasing the agricultural value of the product.

Keywords: Biowaste, Composting, Co-composting, Star Grass, Support material.

\section{Efeito da incorporação de pasto estrela sobre o processo e a qualidade do produto de compostagem de biorresiduos}

\section{Resumo}

A composição predominantemente orgânica dos bioresíduos (BOM) presentes nos resíduos sólidos municipais, potencializa seu aproveitamento através da compostagem; noentanto, apresentam deficiências que podem ser mitigadas com a incorporação de materiais acondicionadores como os materiais de suporte. Neste estudo, foi avaliado o efeito da incorporação de Pasto Estrela (PE) na compostagem de BOM, avaliando quatro proporções B0M:PE (A0-100:00, A1-90:10, A2-80:20, A3-70:30), encontrando-se efeitos favoráveis das misturas respeito ao tratamento controle (A0-100\% BOM). A2 manteve as temperaturas mais altas; A2 e A3 registraram a maior redução de sólidos voláteis (SV) e concentração final de nitrogênio total (NT) e os produtos finais de A2 e A3 também apresentaram melhor qualidade quanto à capacidade de troca catiônica, teor de nutrientes (fósforo, potássio e NT), densidade aparente, capacidade de retenção de umidade e teor de matéria orgânica, sendo o produto de A2 o de maior valor agronômico, segundo a Norma Técnica Colombiana. Proporções menores ou iguais às de A1, não afetam significativamente o processo e a qualidade do produto final e proporções maiores que A3 podem favorecer a perda de nitrogênio devido ao aumento de porosidade, diminuindo assim o valor agrícola do produto.

Palavras chave: Bioresíduos, Compostagem, Co-compostagem, Material de suporte, Pasto Estrela.

\section{Abreviaciones}

A0: Tratamiento control BOM:PE - 100:00

A1: Tratamiento BOM:PE - 90:10

A2: Tratamiento BOM:PE - 80:20

A3: Tratamiento BOM:PE - 70:30

ANOVA: Análisis de Varianza
BOM: Biorresiduos de Origen Municipal

CE: Conductividad Eléctrica

CIC: Capacidad de Intercambio Catiónico

C/N: Carbón/Nitrógeno

CF: Coliformes Fecales

COT: Carbono Orgánico Total 
CT: Coliformes Totales

KT: Potasio Total

KOH: Hidróxido de Potasio

MA: Materiales Acondicionadores

ME: Materiales de Enmienda

MO: Materia Orgánica

MS: Materiales de Soporte

NCh: Normas Chilenas Aprobadas

NT: Nitrógeno Total

NTC: Norma Técnica Colombiana

PE: Pasto Estrella

PT: Fósforo Total

RSM: Residuos Sólidos Municipales

SV: Sólidos Volátiles

\section{Introducción}

Los biorresiduos de origen municipal (BOM) representan la mayor fracción de los residuos sólidos municipales ( $>50 \%$ ) en países en desarrollo (Zhang et al., 2014; Zhou et al., 2018), cuya producción pasará de 1.300 millones/t-año generados en 2012 a 2200 para el año 2025 (Sukholthaman y Shar, 2014), lo cual supone retos ambientales, económicos y sociales (Papargyropoulou et al., 2014), debido a que más del $90 \%$ de los BOM generados son enviados a disposición final en rellenos sanitarios y/o vertederos con efectos negativos como la generación de gases de efecto invernadero, lixiviados, y daños a la salud pública (Thi et al., 2015).

El compostaje es una alternativa tradicionalmente empleada para el aprovechamiento de BOM, principalmente en países en desarrollo. Sin embargo, aspectos como su composición física heterogénea, alta humedad, conductividad eléctrica y deficiencia de parámetros como carbono orgánico total (COT) y fósforo total (PT) (Faverial et al., 2016; Götze et al., 2016), pueden condicionar el proceso y la calidad del producto final (Nigussie et al., 2017; Oviedo et al., 2017).

Para mejorar el proceso de compostaje de BOM, es habitual incorporar MS para mejorar tanto las características fisicoquímicas de los BOM como para favorecer el desarrollo del proceso y mejorar la calidad del producto final (Bernal et al., 2009). No obstante, si la proporción de mezcla BOM:MS es inadecuada, pueden generarse efectos antagónicos o sinérgicos (Jiang et al., 2011; Zhang y Sun, 2016), por lo que es indispensable definir la mejor proporción.

Entre los MS se encuentran materiales como aserrín, residuos verdes, cascarilla de arroz, paja, entre otros (Li et al., 2013; Soto-Paz et al., 2017). El uso de residuos verdes es ampliamente documentado en la literatura ( $\mathrm{Li}$ et al., 2013). Reyes-Torres et al., (2018) indican que el uso de este residuo puede incrementar el tiempo de proceso debido a la presencia de compuestos lignocelulíticos; no obstante, favorece la formación de sustancias húmicas que incrementan el valor agrícola del producto final. Oviedo et al., (2013 y 2015) evaluaron la incorporación de residuos verdes (i.e Pasto estrella-PE) sobre el compostaje de BOM, encontrando que la proporción de mezcla (17\% y $34 \%$ de PE) incide directamente sobre el proceso y calidad del producto. Para mejorar el compostaje de BOM con residuos verdes, además de evaluar diferentes proporciones de mezcla, es recomendable considerar la aplicación de una frecuencia de volteo constante durante las fases activas del proceso (mesofilica y termofilica) y de enfriamiento, lo que puede contribuir a optimizar el proceso de compostaje y mejorar la calidad del producto final, ampliando sus potenciales usos en la agricultura. Adicionalmente, son limitados los estudios que consideran este aspecto (Soto-Paz et al., 2017).

En este estudio se evaluó simultáneamente a escala piloto, el efecto de la incorporación de PE en diferentes proporciones sobre el mejoramiento del proceso y la calidad del producto final en términos de características agrícolas, del compostaje de BOM procedentes de un municipio que realiza separación en la fuente y recolección selectiva de los residuos sólidos municipales (Oviedo et al., 2017).

\section{Materiales y métodos}

\subsection{Unidades Experimentales}

Se evaluaron cuatro proporciones BOM:PE (A0 100:00; A1 90:10; A2 80:20; A3 70:30, en base húmeda), las cuales fueron definidas considerando como criterios que: (i) los BOM deben ser el sustrato predominante (> $60 \%$ ) y (ii) la relación $\mathrm{C} / \mathrm{N}$ de la mezcla resultante debe ser mayor o igual a 15 (Kumar et al., 2010). Los 
tratamientos se evaluaron por duplicado en pilas con forma cónica de $150 \mathrm{~kg}$, altura entre 0.7 a $0.8 \mathrm{~m}$ (Oviedo et al., 2017) y se contó con un tratamiento control que consistió en una pila de solo BOM (A0 100:00).

El sustrato (BOM) y co-sustrato (PE), se les realizó una reducción del tamaño de partícula entre 4 y $7 \mathrm{~cm}$, acorde con Onwosi et al., (2017). En todos los tratamientos se aplicó una frecuencia de volteo manual de dos veces por semana durante la fase activa (fase termofílica) del proceso y durante la etapa de enfriamiento y maduración, esta se redujo a una vez por semana (Oviedo et al., 2017).

\subsection{Caracterización de sustrato y co- sustrato}

Los BOM fueron obtenidos de una instalación de compostaje localizada en un municipio de Colombia donde se practica separación en la fuente y recolección selectiva (Oviedo et al., 2017). El PE se obtuvo de zonas verdes circundantes a la instalación de compostaje. Se realizó un programa de muestreo y caracterización de BOM (doce muestreos) y PE (cinco muestreos).

Las variables medidas a ambos materiales fueron: humedad (gravimetría) y $\mathrm{pH}$ (potenciometría en un extracto de $50 \mathrm{ml}$ de agua destilada y $10 \mathrm{~g}$ de muestra) acorde con ICONTEC (2011); del mismo extracto se midió conductividad eléctrica (CE) y capacidad de intercambio catiónico (CIC). El contenido de sólidos volátiles (SV) se calculó por el método de calcinación a $550^{\circ} \mathrm{C}$ siguiendo las recomendaciones de Ali et al. (2013). COT fue determinado con los métodos descritos en la Norma Técnica Colombiana (NTC) 5167 (Icontec, 2011). NT se midió de acuerdo a la NTC 370 (Icontec, 1997); mientras que PT se cuantificó por espectrofotometría. El contenido de KT se determinó por absorción atómica. Lignina (\%) y celulosa (\%) de acuerdo con PJ Van Soest y Wine (1967). Adicionalmente, se midió el contenido de coliformes totales (CT) y coliformes fecales (CF) según la NTC 5167. Los datos reportados de los parámetros mencionados, corresponden al valor medio de tres mediciones, con su respectiva desviación estándar.

\subsection{Monitoreo del proceso y de la calidad del producto}

Con relación al monitoreo de las pilas de compostaje, se midió diariamente la temperatura en el centroide y el perímetro de cada pila con un termómetro de carátula de $30 \mathrm{~cm}$ (Termómetro Reotemp, San Diego, California, EE.UU); la temperatura ambiente se registró con una termocupla TC spring loaded. Por otra parte, se midieron el pH, SV, NT y PT (Acosta-Durán et al., 2013; Oviedo-Ocaña et al., 2017) dos veces por semana hasta el inicio de la fase de enfriamiento, donde se redujo la frecuencia a una vez por semana.

Al final del proceso, se realizó un tamizado manual (tamiz de 1,25 cm) de los productos de cada tratamiento, determinando variables como $\mathrm{pH}, \mathrm{CIC}, \mathrm{CE}$, COT, NT, PT, KT (Navia-Cuetia et al., 2013; Bohórquez et al., 2014; Oviedo et al., 2017), además de la estabilidad de los productos en términos del índice respirométrico dinámico (IRD) a $37^{\circ} \mathrm{C}$, cuantificando la concentración de oxígeno con un analizador portátil de gases modelo GFM406 (Reino Unido) (Ponsá et al. (2010).

Tanto a los resultados del proceso como de la calidad de los productos de los diferentes tratamientos, se les realizó un análisis estadístico empleando el software R versión 3.6.5®; para determinar la existencia de diferencias estadísticas significativas $(p<0.05)$ y se realizó un análisis de varianza - ANOVA de una vía y se aplicó la prueba post-ANOVA (Tukey: $p$ $<0.05$ ) para determinar el mejor tratamiento respecto a la variable evaluada.

\section{Resultados y discusión}

\subsection{Caracterización de sustrato y co-} sustrato

La Tabla 1 muestra la caracterización de BOM y PE y de las mezclas evaluadas en los diferentes tratamientos. Con relación a los BOM, el pH ácido está relacionado con el alto contenido de humedad (>70\%) que favorece la producción de ácidos grasos volátiles (Sundberg et al., 2013); se observa además la predominancia orgánica de los BOM y dado que el NT es superior al reportado por autores como Thi et al., (2015) y Campuzano y González-Martínez, (2016), se presenta una baja relación $\mathrm{C} / \mathrm{N}(<21)$. Se observa además una baja concentración de PT $(<1 \%)$ y un elevado contenido de potasio, debido a la presencia de cáscaras de plátano y banano en los BOM (Oviedo et al., 2017). 
Kevin Foronda-Zapata, Cielo Arias-Giraldo, Jonathan Soto-Paz, Luis Fernando Marmolejo-Rebellón, Patricia Torres-Lozada

TABLA 1. CARACTERIZACIÓN DE BOM, PE Y TRATAMIENTOS BOM:PE

\begin{tabular}{c|c|c|c|c|c|c|c|c|c|}
\hline & \multicolumn{7}{|c|}{ Parámetros } & \\
\cline { 2 - 11 } Tratamiento & $\begin{array}{c}\text { Muestras } \\
\left(\mathbf{n}^{\circ}\right)\end{array}$ & $\begin{array}{c}\text { Humedad } \\
(\%)\end{array}$ & $\mathbf{p H}$ & SV (\%) & COT (\%) & NT (\%) & $\begin{array}{c}\text { Relación } \\
\text { C/N }\end{array}$ & PT (\%) & KT (\%) \\
\hline BOM & 12 & $77.3 \pm 2.6$ & $5.5 \pm 0.3$ & $74.3 \pm 0.4$ & $31.0 \pm 2.5$ & $1.5 \pm 0.1$ & $21.4 \pm 1.4$ & $0.4 \pm 0.2$ & $1.8 \pm 0.2$ \\
\hline PE & 5 & $65.1 \pm 4.3$ & $7.5 \pm 0.6$ & $76.1 \pm 8.4$ & $37.8 \pm 6.3$ & $1.6 \pm 0.3$ & $23.1 \pm 0.8$ & $0.5 \pm 0.2$ & $1.4 \pm 0.2$ \\
\hline A1 (90:10) & 5 & $71.8 \pm 1.7$ & $6.2 \pm 0.0$ & $58.7 \pm 4.4$ & $34.9 \pm 1.7$ & $1.5 \pm 0.4$ & $22.5 \pm 0.5$ & $0.5 \pm 0.01$ & $1.6 \pm 0.1$ \\
\hline A2 (80:20) & 5 & $71.1 \pm 0.5$ & $6.9 \pm 0.9$ & $63.6 \pm 6.5$ & $36.9 \pm 4.3$ & $1.4 \pm 0.3$ & $23.5 \pm 0.8$ & $0.5 \pm 0.1$ & $1.5 \pm 0.2$ \\
\hline A3 (70:30) & 5 & $71.3 \pm 5.9$ & $6.9 \pm 0.5$ & $71.4 \pm 3.3$ & $35.2 \pm 4.7$ & $1.2 \pm 0.3$ & $25.1 \pm 1.4$ & $0.7 \pm 0.3$ & $1.8 \pm 0.2$ \\
\hline
\end{tabular}

Celulosa: $1.39 \pm 0.19$ (\%) para BOM y $3.14 \pm 0.93$ para PE\%; Lignina: $0.57 \pm 0.31$ (\%) para BOM y $7.18 \pm 1.28 \%$ para PE; Hemicelulosa: $2.37 \pm 0.15$ para BOM y $6.18 \pm 1.88 \%$ para $\mathrm{PE}$.

Con relación al PE, el valor del pH fue próximo a la neutralidad y mayor al de los BOM, similar a lo reportado en otras investigaciones (Zhang y Sun, 2016; Reyes-Torres et al., 2018). Los valores de la relación $\mathrm{C} / \mathrm{N}$, contenido de PT y KT, se encuentran dentro de los reportados por Vandecasteele et al. (2016) para residuos verdes $(21.5-49.2,0.12-0.21 \%$ y $0.43-1.44 \%$, respectivamente), no obstante el contenido de humedad fue mayor (42.6 - 52.2\%), lo que puede estar asociado a que éstos presentan alta variabilidad por las condiciones climáticas y geográficas de la región (Reyes-Torres et al., 2018) y por el tiempo de cosecha del PE (Chanpla et al., 2017). El contenido de celulosa y lignina fue superior en el PE comparativamente con los BOM.

Respecto a los tratamientos BOM:PE (A1, A2 Y A3), se observa que la incorporación de PE incrementó el pH, SV, COT relación C/N y PT en comparación con los BOM, además de aportar mayor porosidad (Torres et al., 2007), lo que puede beneficiar el inicio del proceso al aumentar la disponibilidad de aire en los tratamientos y estimular la actividad biológica (Waqas et al., 2018).

\subsection{Monitoreo del proceso}

Degradación de la materia orgánica durante el proceso

La Figura 1 muestra los perfiles de temperatura, $\mathrm{SV}$ y $\mathrm{pH}$, en que se observa una tendencia similar en las dos primeras variables, lo que está acorde con De Guardia et al. (2010), quienes indican que la biodegradación de la MO y la temperatura por acción de la actividad microbiana, se correlacionan positivamente.
En todos los experimentos, la mayor reducción en el contenido de SV se observó durante la fase termofílica (ver Figura 1b), que está de acuerdo con la rápida descomposición de compuestos fácilmente degradables como carbohidratos, proteínas que son usados como fuente de energía y nitrógeno por los consorcios microbianos (Jiang-ming, 2017; Hemidat et al., 2018; Waqas et al., 2018). Entre los tratamientos evaluados, la máxima reducción de SV se presentó en A2 y A3, los cuales consistentemente presentaron las mayores temperaturas medias durante el proceso indicando una mayor actividad microbiológica, que pudo estar favorecida por el incremento en la disponibilidad de oxígeno asociada a la porosidad aportada por el PE (Oviedo et al., 2015). En contraste, las menores pendientes de biodegradación se observaron en los tratamientos A0 y A1, los cuales no mostraron diferencias significativas ( $p>0.05$ ).

Acorde con Soobhany (2018), estos tratamientos alcanzaron condiciones de higienización debido a que se lograron temperaturas superiores a $60^{\circ} \mathrm{C}$ durante tres o más días consecutivos y se evidenció que la proporción de mezcla tiene un efecto sinérgico y significativo $(\mathrm{p}=0.012)$ sobre la degradación de la MO. La Figura 1C muestra los perfiles de pH durante el proceso. Conforme la MO fue degradada, se observó un incremento gradual del $\mathrm{pH}$, pasando de ligeramente ácido a alcalino (valores entre 8.0 y 9.5 unidades). Autores como Wang et al., (2015) y Cáceres et al., (2018) atribuyen este comportamiento a la mineralización de compuestos ricos en NT (i.e proteínas, aminoácidos y péptidos) y a nitrógeno amoniacal producido durante el proceso de amonificación. 
Figura 1. Monitoreo del proceso en términos de temperatura, $\mathrm{SV}$ y $\mathrm{pH}$. A perfil de temperaturas. B degradación de la MO (SV). C perfiles de $\mathrm{pH}$

A

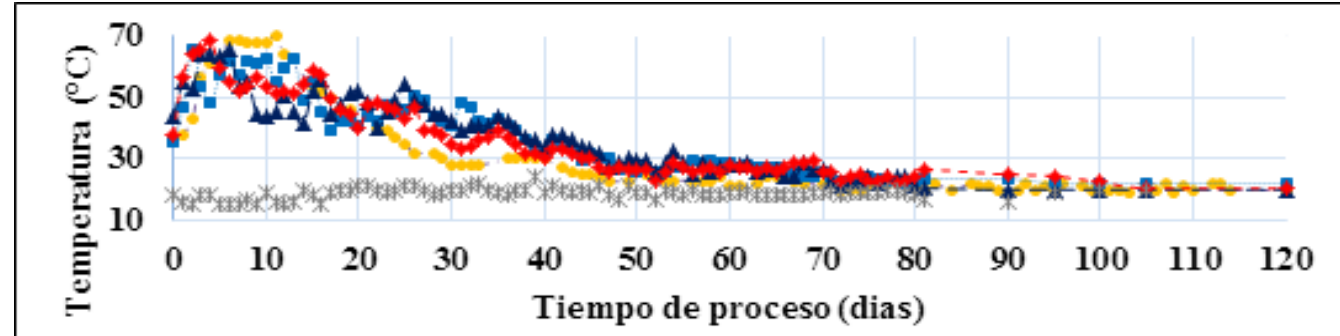

$\ldots$ A0 $\quad$ Al $\rightarrow-A 2 \quad \cdots$ A3 $\quad *$ Tambiente

B

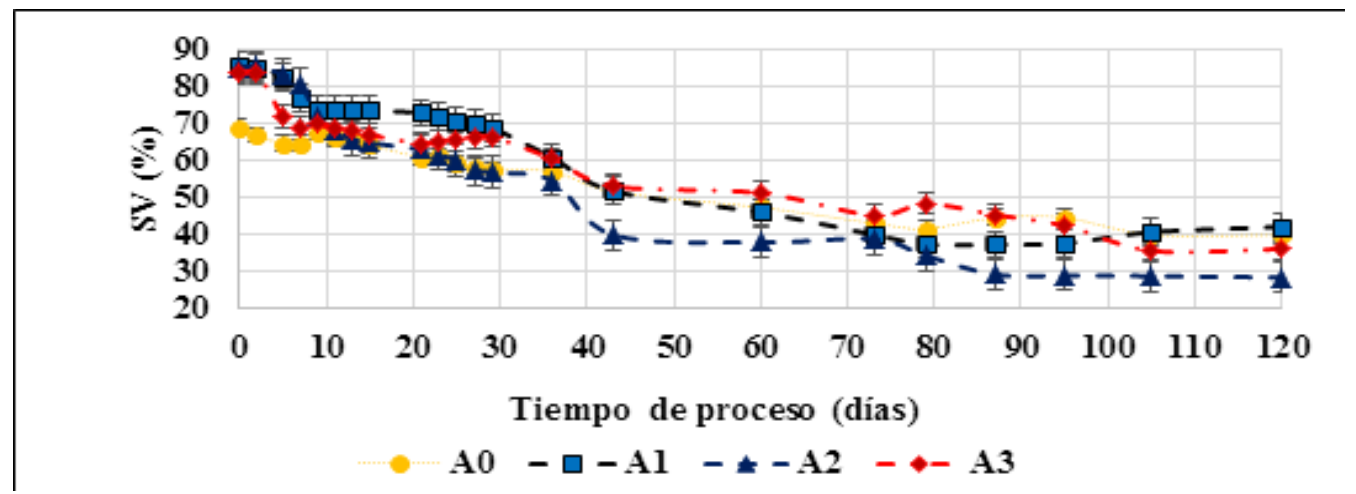

C

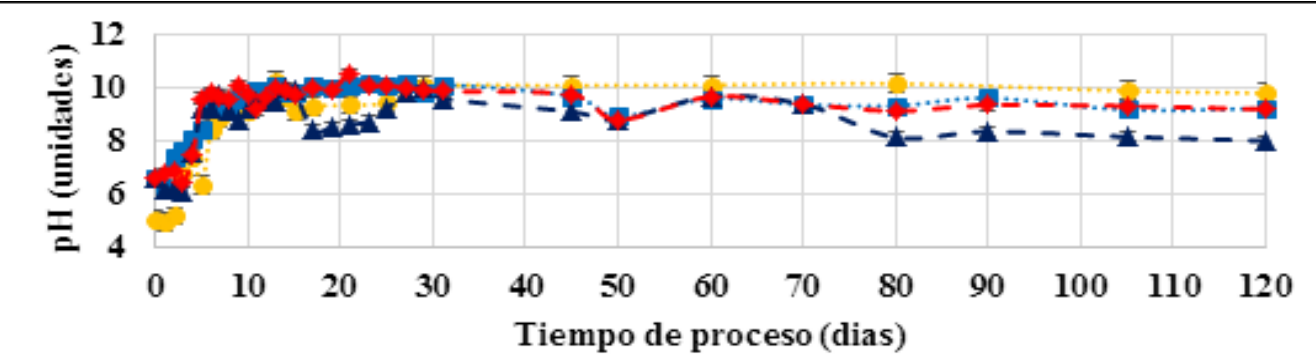

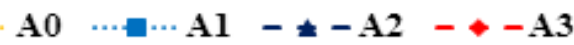

Adicionalmente, como los BOM presentan KT (debido a la presencia de cáscaras de plátano y banano) este elemento, en asociación con el agua presente en la matriz, pudo propiciar la formación de $\mathrm{KOH}$, que es una base fuerte (Kalemelawa et al., 2012) que también pudo contribuir con el incremento del pH. Este comportamiento fue observado también por (Oviedo et al., 2017) en la zona de estudio.
Durante la etapa de enfriamiento (temperatura $<45^{\circ} \mathrm{C}$ y después del día 55), en todos los tratamientos los perfiles de SV tendieron a estabilizarse producto del proceso de biodegradación de compuestos de lenta biodegradación como lignina y hemicelulosa; consistentemente la temperatura decreció hasta alcanzar al final del proceso valores entre 22 y $24^{\circ} \mathrm{C}$. Las mayores temperaturas observadas respecto al control, están asociadas 
con la presencia de compuestos de lenta de biodegradación que prolongan el proceso (mayor proporción de PE) (Haynes et al., 2015), pero contribuyen a incrementar la formación de compuestos húmicos (Zhang y Sun, 2016; Zhou et al., 2018; Martínez-Salgado et al., 2019).

Al final del proceso, todos los tratamientos presentaron valores de $\mathrm{pH}$ alcalinos (A0, A1 y A3 >9 unidades y A2 8.1 unidades), siendo A2 el de mejores valores, ya que está en el rango óptimo (7.0 - 8.5 unidades) según criterios de calidad del compost sugerida por normativas europeas (Cesaro et al., 2015) y por la NTC 5167 ICONTEC (2011).

\section{Monitoreo de nutrientes}

La Figura 2 presenta los perfiles de NT y PT durante el proceso. En la Figura 2A se observa que todos los tratamientos empiezan a disminuir el contenido de NT desde el inicio del proceso, lo que está asociado a la actividad biológica y a la volatilización del nitrógeno por el consecuente aumento de la temperatura y el $\mathrm{pH}$ durante la etapa termofílica, (Oudart et al., 2015). El menor descenso en el contenido de NT se observó en A2 y A3, probablemente asociado a una menor pérdida de amoniaco; al respecto, autores como Zhang y Sun (2016) indican que la presencia de materiales lignocelulósicos en las mezclas, favorece la inmovilización de NT.

Sin embargo, la mayor reducción de NT en A3, se puede deber a una mayor volatilización en forma amoniacal favorecida por el aumento de la porosidad (Cáceres et al., 2018), la cual está relacionada con el incremento en la proporción de PE en la mezcla BOM:PE.

Figura 2. Evolución de los nutrientes durante el proceso. A perfiles de NT. B perfiles de PT

A

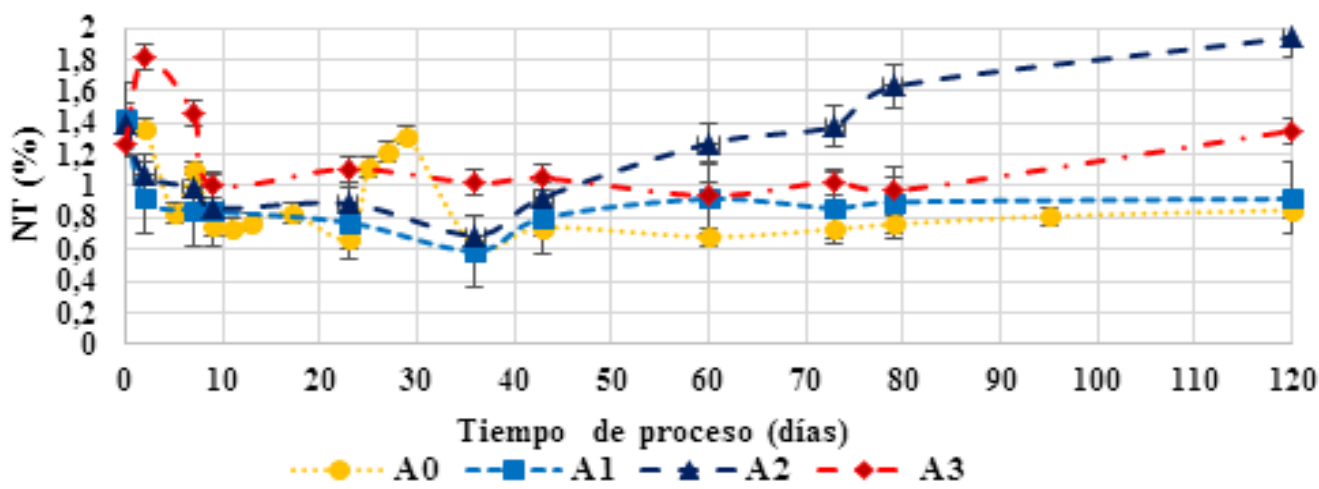

B

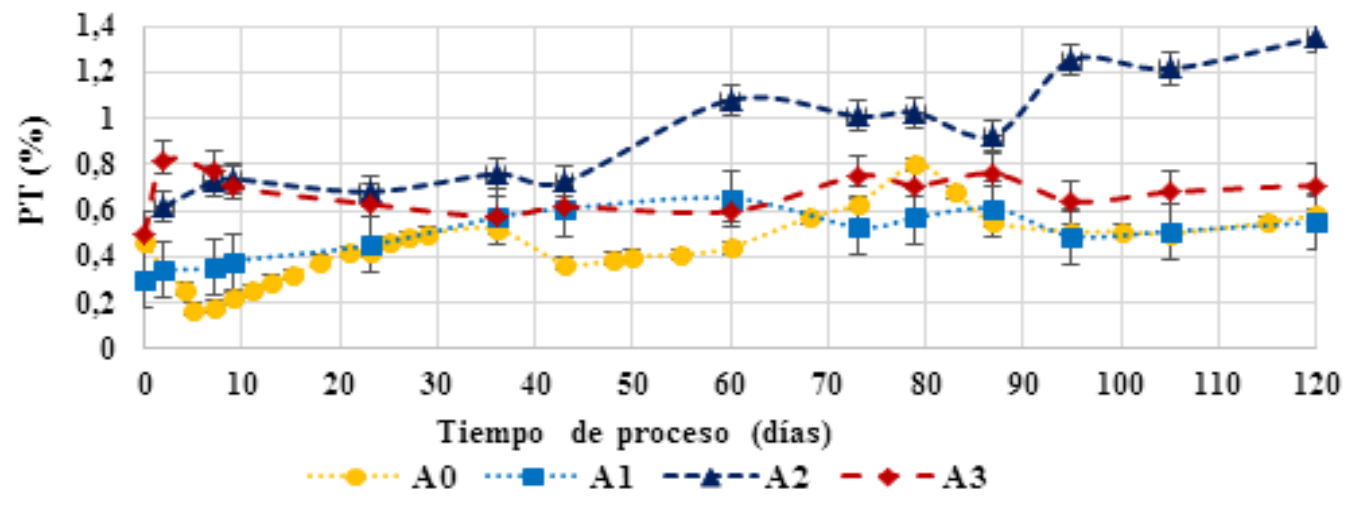


La dinámica del PT presentó un comportamiento fluctuante durante el proceso, lo que guarda relación con las posibles variaciones en la actividad microbiana en respuesta a factores ambientales como temperatura, humedad y disponibilidad de carbono (Parkinson et al., 2004); en general se observa un incremento gradual al final del proceso, que está asociado a la mineralización de la MO (Figura 2B).Igualmente, el PT fue más alto en A2 comparativamente con los tratamientos A1 y A3. El descenso en la concentración de PT al inicio del proceso puede asociarse a la lixiviación del fósforo soluble y a su inmovilización por parte de los microrganismos (Parkinson et al., 2004). Richardson y Simpson (2011) sugieren que la incorporación de residuos verdes puede promover la mineralización del PT durante la biodegradación de la MO.

Estadísticamente, los tratamientos presentaron diferencias significativas $(\mathrm{p}=1.23 \mathrm{e}-8)$ y la prueba de Tuckey determinó que con excepción de los tratamientos A0 y A1 ( $p=0.97)$, todos los tratamientos presentaron diferencias significativas entre sí; A2 y A3 presentaron el mayor contenido de PT (A3 - A2, p= 0,004). Los valores encontrados en este estudio muestran una mejora respecto a otros autores en el compostaje de BOM con residuos verdes (Reyes-Torres et al., 2018).

\subsection{Callidad del producto final}

La Tabla 2 muestra los resultados de la calidad fisicoquímica del producto final de cada tratamiento evaluado y su comparación con los requerimientos para abono orgánico estipulados por la norma nacional (NTC $5167,2011)$. Con respecto al $\mathrm{pH}$, los productos presentaron valores con tendencia a ser alcalinos (i.e $\mathrm{pH}>7.5$ ), aunque cumplen con lo estipulado en la normativa nacional (NTC 5167, 2011). Acorde con Lasaridi et al. (2006), el $\mathrm{pH}$ del producto debe fluctuar entre 6.0 y 8.5 para que el proceso se pueda aplicar a una amplia variedad de plantas, por lo que los tratamientos A2 y A3 muestran mayor potencial para estimular la actividad vegetal.

En cuanto a la CIC, todos los tratamientos presentaron valores mayores a $20 \mathrm{meq} / 100 \mathrm{~g}$, indicando que estimularían la actividad biológica por el intercambio de bases con el suelo. Los valores de CE son bajos en comparación del límite propuesto en las normativas e indica poca presencia de sales. Acorde con Lasaridi et al. (2006) esto es importante para reducir problemas de fitotoxicidad en las plantas.

Con respecto al contenido de COT, NKT, PT y KT el tratamiento A2 tiene el mayor potencial para el uso agrícola, ya que presenta mejor contenido de nutrientes y mejores características agronómicas como CIC y CE. Respecto a la calidad biológica, todos los tratamientos con PE presentaron condiciones de estabilidad debido a que tuvieron un consumo de oxígeno inferior a $1.0 \mathrm{mgO} 2$ gSV $^{-1}$ (Barrena et al., 2006). Los productos más estables se presentaron en A2 y A3, mostrando el efecto sinérgico de incrementar la proporción de mezcla.

TABLA 2. CALIDAD FISICOQUÍMICA DEL PRODUCTO FINAL

\begin{tabular}{|c|c|c|c|c|c|c|}
\hline Parámetro & Unidad & $\begin{array}{l}\text { Pila Control } \\
\text { (BOM) }\end{array}$ & $\begin{array}{c}\text { A1 } \\
(90: 10)\end{array}$ & $\begin{array}{c}\text { A2 } \\
(80: 20)\end{array}$ & $\begin{array}{c}\mathbf{A 3} \\
(70: 30)\end{array}$ & NTC 5167 \\
\hline $\mathrm{pH}$ & Unidades & $8.9 \pm 0.5$ & $8.5 \pm 0.1$ & $8.1 \pm 0.1$ & $7.8 \pm 0.3$ & $4.0-9.0$ \\
\hline $\mathrm{CIC}$ & meq/100g & $22.4 \pm 2.2$ & $52.6 \pm 1.8$ & $65.6 \pm 2.1$ & $59.6 \pm 2.8$ & $\geq 30$ \\
\hline CE & $\mathrm{dS} / \mathrm{m}$ & $0.4 \pm 0.1$ & $1.3 \pm 0.0$ & $0.4 \pm 0.0$ & $0.7 \pm 0.02$ & - \\
\hline NT & $\%$ & $0.8 \pm 0.3$ & $1.0 \pm 0.3$ & $2.3 \pm 0.2$ & $1.8 \pm 0.1$ & $>1.0$ \\
\hline COT & $\%$ & $12.8 \pm 2.4$ & $10.8 \pm 0.9$ & $20.3 \pm 0.4$ & $23.1 \pm 1.4$ & $\geq 15$ \\
\hline PT & $\%$ & $0.6 \pm 0.3$ & $1.1 \pm 0.2$ & $1.4 \pm 0.1$ & $1.2 \pm 0.1$ & $>1.0$ \\
\hline $\mathrm{KT}$ & $\%$ & $2.2 \pm 1.2$ & $2.1 \pm 0.1$ & $2.1 \pm 0.1$ & $2.3 \pm 0.3$ & $>1.0$ \\
\hline Estabilidad & $\mathrm{mgO}_{2} / \mathrm{gSVh}$ & $0.9 \pm 0.1$ & $0.77 \pm 0.1$ & $0.65 \pm 0.1$ & $0.66 \pm 0.1$ & - \\
\hline $\mathrm{CF}$ & $\mathrm{NMP} / \mathrm{g}$ & $35.0 \pm 3$ & nd & nd & nd & $<1000$ \\
\hline $\mathrm{CT}$ & $\mathrm{NMP} / \mathrm{g}$ & $59.0 \pm 11$ & $35.3 \pm 1.4$ & $31.9 \pm 2.2$ & $29.0 \pm 3.9$ & $<1000$ \\
\hline
\end{tabular}


Respecto a las características microbiológicas, todos los productos presentaron valores inferiores al límite máximo de CF (<1000 NMP/g) fijado por la NTC 5167 (2011); sin embargo, los tratamientos con incorporación de PE presentaron valores inferiores respecto al tratamiento control A0 (100\% BOM) que fue el producto que reportó los valores más altos de CT y CF.

\section{Conclusiones}

Los resultados del estudio indicaron que el tratamiento A1 (BOM:PE, 90:10) no tuvo un efecto significativo sobre el proceso y calidad del producto final respecto a la unidad control de solo BOM (A0). Por otro lado, el tratamiento A3 (BOM:PE, 70:30) aunque mostró un mejor desempeño durante el proceso y mejor calidad del producto final que A0, presentó una mayor reducción de nitrógeno total que A2 (BOM:PE, 80:20). Por lo anterior, no se recomendaría la incorporación de proporción de PE menores o iguales a $10 \%$ debido a su poca incidencia sobre el proceso de compostaje de BOM.

Entre las mezclas BOM:PE evaluadas, la proporción 80:20 (A2) fue la más favorable, con valores de pH más cercanos a la neutralidad, inicio más rápido de la fase termofílica y mayor reducción de sólidos volátiles durante el proceso respecto a los otros tratamientos. En cuanto a la calidad del producto final, también fue el tratamiento que mejor se ajustó a la norma técnica colombiana para productos para la industria agrícola, productos orgánicos usados como abonos o fertilizantes y enmiendas de suelo.

\section{Agradecimientos}

Los autores agradecen el apoyo financiero de la Universidad del Valle por la financiación del Proyecto C.I. 2985 y a COLCIENCIAS por el apoyo a la formación doctoral del candidato Ing. Jonathan Soto-Paz a través de la Convocatoria 727-2015.

\section{Referencias}

Acosta-Durán, C. M.; Solís-Pérez, O.;Villegas-Torres, 0. G.; Cardoso-Vigueros, L. (2013). Precomposteo de residuos orgánicos y su efecto En la dinámica poblacional de einsenia foetida. Agronomía Costarricense, 37 (1), pp. 127-139.
Ali, U.; Khalid, A.; Mahmood, T; Aziz, I. (2013). Accelerated Biodegradation of Solid Organic Waste through Biostimulation. Proceedings of the Pakistan Academy of Sciences, 50 (1), pp. 37-46.

Barrena, R.; Vázquez, F.; Sánchez, A. (2006). The use of respiration indices in the composting process: a review. Waste Management \& Research, 24 (1), pp. 24-37. Doi:10.1177 / $0734242 X 06062385$

Bernal, M. P.; Alburquerque; J. A.; Moral, R. (2009). Composting of animal manures and chemical criteria for compost maturity assessment. A review. Bioresource technology, 100 (22), pp. 5444-5453. Doi: 10.1016/j. biortech.2008.11.027

Bohórquez, A.; Puentes, Y.; Menjivar, J. C. (2014). Evaluación de la calidad del compost producido a partir de subproductos agroindustriales de caña de azúcar. Corpoica Ciencia y Tecnología Agropecuaria, 15 (1), pp 73-81

Cáceres, R., Malińska, K. y Marfà, O. (2018). Nitrification within composting: A review. Waste Management, 72, pp. 119-137. Doi: 10.1016/j.wasman.2017.10.049

Chanpla, M.; Kullavanijaya, P.; Janejadkarn, A.; Chavalparit, 0. (2017) Effect of harvesting age and performance evaluation on biogasification from Napier grass in separated stages process. KSCE Journal of Civil Engineering, pp. 1-6. Doi: 10.1007/s12205-017-1164-y

Campuzano, R.; González-Martínez, S. (2016). Characteristics of the organic fraction of municipal solid waste and methane production: A review. Waste Management, pp. 54: 3-12. Doi: 10.1016/j.wasman.2016.05.016

Cesaro, A.; Belgiorno, V.; Guida, M. (2015). Compost from organic solid waste: Quality assessment and European regulations for its sustainable use. Resources, Conservation and Recycling ,94(0), pp. 72-79. Doi: 10.1016/j.resconrec.2014.11.003

De Guardia, A.; Mallard, P.; Teglia, C.; Marin, A.; Le Pape, C.; Launay, M.; Benoist, J. C.; Petiot, C. (2010). Comparison of five organic wastes regarding their behaviour during composting: Part 1, biodegradability, stabilization kinetics and temperature rise. Waste Management, 30(3), pp. 402-414. Doi: 10.1016/j.wasman.2009.10.019

Faverial, J.; Boval, M.; Sierra, J.; Sauvant, D. (2016). End-product quality of composts produced under tropical and temperate climates using different raw materials: A meta-analysis. Journal of Environmental Management, 183, pp. 909-916. Doi: 10.1016 / j.jenvman.2016.09.057 
Götze, R.; Boldrin, A.; Scheutz, C.; Astrup, T. F. (2016). Physico-chemical characterisation of material fractions in household waste: Overview of data in literature. Waste Management, 49, pp. 3-14. Doi: 10.1016 / j.wasman.2016.01.008

Haynes, R.J.; Belyaeva, O. N.; Zhou, Y. F. (2015). Particle size fractionation as a method for characterizing the nutrient content of municipal green waste used for composting. Waste Management, 35, pp. 48-54. Doi: 10.1016/j.wasman.2014.10.002

Hemidat, S.; Jaar, M.; Nassour, A.; Nelles, M. (2018). Monitoring of Composting Process Parameters: A Case Study in Jordan. Waste and Biomass Valorization, 9(12), pp. 2257-2274. Doi: 10.1007 / s12649-0180197-x

ICONTEC (2011). Norma Técnica Colombiana 5167. Productos para la Industria Agrícola, Productos Orgánicos Usados como Abonos o Fertilizantes y Enmiendas de Suelo.

Jiang T., Schuchardt F., Li G., Guo R. y Zhao Y. (2011). Effect of $\mathrm{C} / \mathrm{N}$ ratio, aeration rate and moisture content on ammonia and greenhouse gas emission during the composting. Journal of Environmental Sciences, 23 (10), 1754-1760. Doi: 10.1016/S1001-0742(10)60591-8

J Jiang-Ming, Z. (2017). Effect of turning frequency on cocomposting pig manure and fungus residue. Journal of the Air \& Waste Management Association, 67 (3), 313-321. Doi: 10.1080 / 10962247.2016.1232666

Kalemelawa, F., Nishihara, E., Endo, T., Ahmad, Z., Yeasmin, R., Tenywa, M. M. y Yamamoto, S. (2012). An evaluation of aerobic and anaerobic composting of banana peels treated with different inoculums for soil nutrient replenishment. Bioresource Technology, 126, 375-382. Doi: 10.1016/j.biortech.2012.04.030

Kumar, M., Ou, Y. y Lin, J. (2010). Co-composting of green and food waste at low $\mathrm{C} / \mathrm{N}$ ratio. Waste Management, 30(4), 602-609. Doi: 10.1016/j.wasman.2009.11.023

Lasaridi, K., Protopapa, I., Kotsou, M., Pilidis, G., Manios, T. y Kyriacou, A. (2006). Quality assessment of composts in the Greek market: The need for standards and quality assurance. Journal of Environmental Management, 80 (1), 58-65. Doi: 10.1016 / j.jenvman.2005.08.011

Li, Z.; Lu, H.; Ren, L.; He, L. (2013). Experimental and modeling approaches for food waste composting: A review. Chemosphere, 93(7), pp. 1247-1257. Doi: 10.1016/j.chemosphere.2013.06.064

Martínez-Salgado, M.M.; Ortega-Blu, R.; Janssens, M.; Fincheira, P. (2019). Grape pomace compost as a source of organic matter: Evolution of quality parameters to evaluate maturity and stability. Journal of Cleaner
Production, 216, pp. 56-63. Doi: doi.org/10.1016/j. jclepro.2019.01.156

Navia-Cuetia, C. A.; Zemanate-Cordoba, Y.; MoralesVelasco, S.; Alonso Prado, F.; Albán López, N. (2013). Evaluation of different formulations From waste composting crop tomato (solanum lycopersicum). Biotecnología en el Sector Agropecuario y Agroindustrial, 2, pp. 165 - 173.

NCh -Norma chilena de compost 2880- 2004. (2015). Compost - Clasificación y requisitos, 23. Santiago de Chile, 27.

Nigussie, A., Bruun, S., Kuyper, T. W. y De Neergaard, A. (2017). Delayed addition of nitrogen-rich substrates during composting of municipal waste: Effects on nitrogen loss, greenhouse gas emissions and compost stability. Chemosphere, 166, 352-362. Doi: 10.1016/j. chemosphere.2016.09.123

Onwosi, C. O., Igbokwe, V. C., Odimba, J. N., Eke, I. E., Nwankwoala, M. O., Iroh, I. N. y Ezeogu, L. I. (2017). Composting technology in waste stabilization: On the methods, challenges and future prospects. Journal of Environmental Management, 190, 140-157. Doi: 10.1016/j.jenvman.2016.12.051

Oudart, D., Robin, P., Paillat, J.-M. y Paul, E. J. W. M. 2015. Modelling nitrogen and carbon interactions in composting of animal manure in naturally aerated piles. 46, 588-598. Doi: 10.1016/j.wasman.2015.07.044

Oviedo-Ocaña, E. R., Daza Torres, M., Marmolejo Rebellón, L., Osorio Ortiz, Á. y Torres Lozada, P. J. R. C. I. y. D. (2013). Influencia de la incorporación de pasto estrella como material de soporte (Cynodon Plectostachyus) en el compostaje de biorresiduos de origen municipal.Revista Científica Ingeniería y Desarrollo, 31(2), 251-271.

Oviedo-Ocaña, R., Marmolejo-Rebellón, L. F., TorresLozada, P., Daza, M., Andrade, M., Torres-López, W. A. y Abonia-Gonzalez, (2015). Effect of adding bulking materials over the composting process of municipal solid biowastes. Chilean journal of agricultural research, 75(4): 472-480.

Oviedo, R.; Marmolejo, L.; Torres, P. (2017). Advances in research on biowaste composting in small municipalities of developing countries. Lessons from Colombia. Revista Ingenieria Investigacion y Tecnologia, 18(01), pp. 31-42.

Parkinson, R., Gibbs, P., Burchett, S. y Misselbrook, T. (2004). Effect of turning regime and seasonal weather conditions on nitrogen and phosphorus losses during aerobic composting of cattle manure. Bioresource Technology, 91 (2), 171-178. Doi: 10.1016/ S0960-8524(03)00174-3 
Papargyropoulou E, Lozano R, K. Steinberger J, Wright N, Ujang Z Bin (2014). The food waste hierarchy as a framework for the management of food surplus and food waste. Journal Clean Production., 76, 106-115. Doi: https://doi.org/10.1016/j.jclepro.2014.04.020

Ponsá, S., Gea, T. y Sánchez, A. (2010). Different Indices to Express Biodegradability in Organic Solid Wastes. Waste Managament, 39 (2), 706-712. Doi: 10.2134 / jeq2009.0294

Reyes-Torres, M., Oviedo-Ocaña, E. R., Dominguez, I., Komilis, D. y Sánchez, A. (2018). A systematic review on the composting of green waste: Feedstock quality and optimization strategies. Waste Management, 77, 486-499. Doi: 10.1016/j.wasman.2018.04.037

Richardson, A. E. y Simpson, R. J. (2011). Soil microorganisms mediating phosphorus availability update on microbial phosphorus. Plant Physiol, 156 (3), 989-996. Doi: 10.1104 / pp.111.175448

Soobhany, N. (2018). Assessing the physicochemical properties and quality parameters during composting of different organic constituents of Municipal Solid Waste.Journal of Environmental Chemical Engineering, 6 (2), 1979-1988. Doi: 10.1016/j.jece.2018.02.049

Soto-Paz, J., Oviedo-Ocaña, R., Marmolejo-Rebellón, L. F. y Manyoma-Velásquez, P. C. (2017). Compostaje de biorresiduos: Tendencias de investigación y pertinencia en países en desarrollo. DYNA, 84(203), pp. 334-342. Doi: 10.15446/dyna.v84n203.61549

Sundberg, C., Yu, D., Franke-Whittle, I., Kauppi, S., Smårs, S., Insam, H., Romantschuk, M. y Jönsson, H. (2013). Effects of $\mathrm{pH}$ and microbial composition on odour in food waste composting. Waste Management, 33 (1), 204-211. Doi: 10.1016/j.wasman.2012.09.017

Thi, N., Kumar, G. y Lin, C.Y. (2015). An overview of food waste management in developing countries: Current status and future perspective. Journal of Environmental Management, 157, 220-229. Doi: 10.1016/j. jenvman.2015.04.022

Torres, P.; Imery, R.; Perez, A.; Uribe, I. E.; Escobar Rivera, J. C. (2007). Compostaje de biosólidos de Plantas de Tratamiento de Aguas Residuales. Engenharia Agricola, 27 (1), pp.267 - 275. Doi: 10.1590 / S010069162007000100021

Vandecasteele, B., Boogaerts, C. y Vandaele, E. (2016). Combining woody biomass for combustion with green waste composting: Effect of removal of woody biomass on compost quality. Waste Management, 58, 169-180. Doi: 10.1016/j.wasman.2016.09.012
Van Soest, PJ.; Wine, R.; (1967). Uso de detergentes en el análisis de alimentos fibrosos. IV. Determinación de permanganato. Assoc. Oficial Anal. Chem 50(1): 6.

Wang, K., Li, W., Li, X., Ren, N., 2015. Spatial nitrifications of microbial processes during composting of swine, cow and chicken manure. Sci. Rep. 5 (14932), 2015. https://doi.org/10.1038/srep14932.

Waqas, M., Nizami, A. S., Aburiazaiza, A. S., Barakat, M. A., Rashid, M. I. y Ismail, I. M. I. (2018). Optimizing the process of food waste compost and valorizing its applications: A case study of Saudi Arabia. Journal of Cleaner Production, 176, 426-438. Doi: 10.1016/j. jclepro.2017.12.165

Zhang, L. y Sun, X. (2016). Influence of bulking agents on physical, chemical, and microbiological properties during the two-stage composting of green waste. Waste Management, 48, 115-126. Doi: 10.1016/j.wasman.2015.11.032

Zhang C, Su H, Baeyens J, Tan T. (2014). Reviewing the anaerobic digestion of food waste for biogas production. Renew Sustain Energy Rev. 38, 383-392. Doi: https:// doi.org/10.1016/j.rser.2014.05.038

Zhou, H., Zhao, Y., Yang, H., Zhu, L., Cai, B., Luo, S., Cao, J. y Wei, Z. (2018). Transformation of organic nitrogen fractions with different molecular weights during different organic wastes composting. Bioresource Technology, 262, 221-228. Doi: 10.1016 / j.biortech.2018.04.088.

Zhou M, Yan B, Wong JWC, Zhang Y. (2018). Enhanced volatile fatty acids production from anaerobic fermentation of food waste: A mini-review focusing on acidogenic metabolic pathways. Bioresource Technology, 48, 68-78. Doi: https://doi.org/10.1016/j. biortech.2017.06.121

\section{PARA CITAR ESTE ARTÍCULO / TO REFERENCE THIS ARTICLE / PARA CITAR ESTE ARTIGO /}

Foronda-Zapata, K.; Arias-Giraldo, C.; Soto-Paz, J.; Marmolejo-Rebellón, L.F. Torres-Lozada, P. (2020). Efecto de la incorporación de pasto estrella sobre el mejoramiento del proceso y la calidad del producto del compostaje de biorresiduos. Revista EIA, 17(33) enerojunio, Reia33011 pág. 1-11. Disponible en: https://doi. org/10.24050/reia.v17i33.1352 